\title{
Deep Squat - Should We Be Afraid?
}

\author{
Petr Schlegel, Adrián Agricola, Dana Fialová
}

\begin{abstract}
Department of Physical Education and Sports, Faculty of Education, University of Hradec Králové, Czech Republic
\end{abstract}

\begin{abstract}
Squats are among the basic exercises that have become an integral part of exercise programs. It is commonly used by athletes in many sports. Its potential is shown in the development of strength, power, and overall sports performance. The aim of the study was to verify inconsistent views on deep squats, technical design, and related health risks. For the review, the terms "squat, knee, biomechanics, deep, patellofemoral, back squat, performance, arthritis, health, injury, risk" were used, for searching were used databases Web of Science, PubMed, Springer, and Scopus. It turns out that the technique of performing a deep squat has its strict principles, which must be respected and which have a significant impact on the benefits and risks. From loading, the lower position represents an increase in compressive and shear forces on the patellofemoral joint. However, this is a natural state that is not risky. Health hazards cannot be based on analytical-mathematical models, which are insufficient in this aspect. Extreme long-term exposure carries the risk of permanent consequences in the form of osteoarthritis. For prevention or recovery, it is possible to use effective aids like sleeves, kinesio taping, bandages or "knee savers".
\end{abstract}

Key words: training, performance, knee, patellofemoral

\section{INTRODUCTION}

The inclusion of squats among the basic exercises has a rich history. In modern history, it can be found in Die deutsche Turnkunst (Jahn, 1816), where a variant is used where the exerciser is on tiptoe and performs the full range of motion. This variant was dominant and commonly used at the beginning of the 20th century when it was recommended by personalities such as Eugen Sandow (Sandow, 1894) or George Hackenschnidt (Todd \& Hemme, 2013). At the same time, they practiced with different loads, which were held in the hands or placed on the shoulders. With the execution of a squat with the whole feet on the ground, already mentioned by Beaujeu (1828) and also recommended a variant on one leg.

The deep (back) squat with whole feet on the ground and a large barbell placed behind the neck probably originated thanks to Heinrich Steinborn (the 1920s), who was the first to publicly demonstrate it. He lifted the barbell without help from the ground himself - first he leaned over and then squatted. In this way, he could squat with almost 240kg (Giessing \& Todd, 2005). The practice gained popularity and spread rapidly. For its functionality, the squat started to be included in bodybuilding, weightlifting, and other power training sessions. Roughly, in the 1950s, it became their normal part.

Since the 1960s, there have also been critical views on the proper use of deep squats. Studies support these views, experts in the fields (Klein, 1961) and are becoming known to the general sporting public. Some opinions have persisted to this day, and in the area of sports there is no clear, unified view of deep squats (Escamilla et al., 2009). 
Squats are often used by athletes for strength training, compensation or rehabilitation exercises (Schoenfeld, 2010). It is also applied to the general public to develop fitness. It is therefore important to know the advantages and risks of this exercise. The (deep) squat will be understood in the text as a variant where the knee has an angle $<90^{\circ}$ (below parallel) in the lower position. The aim of the study was to evaluate different views on performing a deep squat, to analyze the optimal technical design, and to evaluate the possible risks in its use.

\section{METHODS}

The authors performed a literature review of available human studies on the research topic describing frequently discussed topics in the sports world - a deep squat. The research studies were selected on the basis of research topics such as "squat, knee, biomechanics, deep, patellofemoral, back squat, performance, arthritis, health, injury, risk" found in databases Web of Science, PubMed, Springer, and Scopus. The terms used were searched using AND to combine the keywords listed and using OR to remove search duplication where possible. The end of the search period is limited by February 2020. The quality of the studies was assessed independently by at least two authors, the subjective evaluation was guided by PEDro scale guidelines. Only studies with full agreement were included.

The inclusion criteria were as follows: the period of the publishing of the article was limited by February 2020; only reviewed full-text studies in scientific journals or relevant books were included. The exclusion criteria involved: specific target groups - children, seniors, diseased persons. Figure 1 below then illustrates the selection procedure.

\section{RESULTS AND DISCUSSION}

Altogether 3,383 were identified in all these databases. After removing duplicates and titles/ abstracts unrelated to the research topic, 262 studies remained. Of these, only 44 articles were relevant to the research topic. These research studies were classified according to their relevancy (Fig. 1). The information found in the selected studies on the deep squat is described and discussed in the following sections.

\section{Optimal squat technique}

The squat is a complex movement that can be divided into three parts (Kritz et al., 2009; Myer et al., 2014): 1. starting position, 2. eccentric/descending phase, 3. concentric/ascending phase. The starting position is usually associated with the correct alignment of the body and the optimal settlement of the external load. The main task is to activate all important muscle groups and to take a position complemented by quality inhalation, creating the necessary tension in the center of the body. The descending phase aims to maintain tension throughout the body as well as the fluency of movement up to the limit point at the bottom. The transition to the ascending phase must be controlled without loss of tension throughout the whole body. When moving upwards, it is necessary to maintain the position of all body segments, there is also exhalation. The squat ends in the starting position (Krause et al., 2015; Ramirez et al., 2018).

Correct technical performance is crucial to getting the benefits of squatting (Schoenfeld et al., 2010; Yavuz \& Edgar, 2017). On the contrary, deviations are associated with the risk of overloading and accidents (Fry et al. 2003, Hartmann \& Wirth, 2014). The entire movement must be controlled. Attached Table 1 shows body parts that must be monitored and adjusted optimally. The description is valid for all three movement parts. 
Figure 1. An overview of the selection procedure.
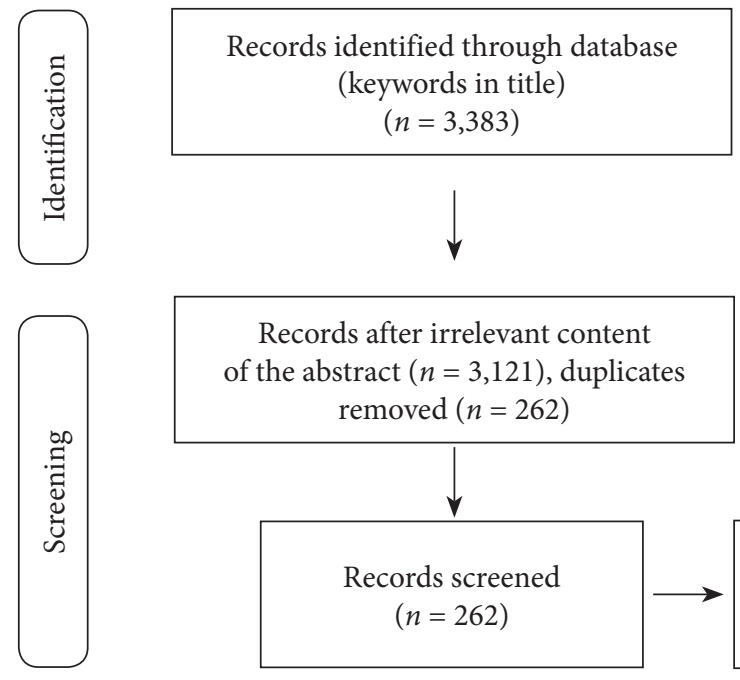

Records after irrelevant content of the abstract $(n=3,121)$, duplicates removed $(n=262)$

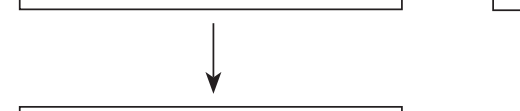

Records excluded, irrelevant to topic $(n=186)$

Full-text articles assessed for eligibility $(n=76)$

Studies included in qualitative synthesis $(n=44)$

Table 1. Optimal squat kinematic consideration (Kritz et al., 2009; Myer et al., 2014).

\begin{tabular}{lll}
\hline Body part & Position & Viewing position \\
\hline Head & neutral (to slight extension) & lateral, anterior \\
Thoracic spine & slightly extended and held rigid & lateral \\
Lumbar spine & neutral & lateral \\
Hip joints & flexed and aligned, neutral pelvic tilt, symmetric, & posterior, anterior \\
& slightly external rotated & \\
Knees & aligned with feet & anterior \\
Tibial angle & adequate, unrestricted & lateral \\
Ankles & neutral & anterior, posterior \\
Foot & flat, stable on the ground & anterior, lateral, posterior \\
\hline
\end{tabular}




\section{Biomechanical analysis}

There are many variations of squats. However, the deep squat is most commonly used in Olympic weightlifting or powerlifting, when the upper pelvis reaches below the patella level as it moves down. Other common variants are partial squat and half squat, i.e., $40^{\circ}$ resp. $70-100^{\circ}$ in the knee joint (Schoenfeld, 2010). It can often be said that the squat, i.e., $100^{\circ}$ or more, is not safe for the knee joint (Escamilla et al., 2008) and is overloaded, negatively affecting knee stability and soft tissue. It is true that when squatting, there is really a greater load on the soft structures of the knee. At various stages of the movement, there is an increase in shear forces (Escamilla, 2001), which must be taken into account by means of a suitable performance. The highest anterior cruciate ligament load (ACL) occurs in flexion of $15-30^{\circ}$ and decreases significantly after exceeding $60^{\circ}$ (Li et al., 2004). In contrast, the posterior cruciate ligament (PCL) is the most heavily loaded in the $90^{\circ}$ flexion (Logterman et al., 2018) and has a greater force on it when moving down (up to $40 \%$ ) (Escamilla et al., 2009). Both ACL and PCL are important for the knee stability, while ensuring optimal transmission of lower limb muscle strength to the joint (Cox \& Bordoni, 2019). With greater flexion, the load on both ligaments decreases (Hehne, 1990) and in the context of the above, it is not necessary to worry about the increased range of motion. Conversely, the study of the difference between weightlifters and the control group regarding PCL and ACL found both ligament hypertrophy in favour of weightlifters (Grzelak et al., 2012).

The technical performance of the squat has a significant effect on the forces acting on the ankle, knee or hip (Wilk et al., 1996). Maximum compression forces are monitored around $90^{\circ}$ flexion. In the course of the squat, the quadriceps, gluteal muscles and hamstrings are dominant. The measured EMG values show their highest activity in the range of $80-100^{\circ}$ (Yavuz \& Erdag, 2017). The correct activation of hamstrings during squatting relieves tension in ACL (Biscarini et al., 2014). Foot position, resp. external rotation, in the hip together with the position of the torso and the load have a significant effect on the activity of the involved muscles and also the transfer of forces to the musculoskeletal system. In this respect, the particular performance has a significant impact (posture width, torso slope, downward movement speed, knee anterior-posterior position, or external load position) (Contreras et al., 2015; Fuglsang et al., 2017; Kushner et al., 2015; Walsh et al., 2007). In addition, the position of the foot or resp. heel elevation compared to flatbed on ACL (Toutoungi et al., 2000), both eccentric (26 vs 95N) and concentric phase (28 vs 49N).

\section{Controversies about squatting}

The first blow for a deep squat was the 1959 research by Dr. Klein. He tried to confirm the hypothesis that the knee ligament was stretched too deeply due to a deep squat, and therefore there is mobility (instability) in the knee (Klein, 1959). The results confirmed this, although from today's point of view the study had major shortcomings. One of the great limitations was the absence of double blindness. Another important limitation was the testing of passive joint mobility. Bill Star, who was part of the research, said that when he was tested, he was extremely pressing on his knee to a great pain (Todd, 1984). Two years later, there was the second blow for the deep squat, made again by Klein (Klein, 1961). By being a recognized authority, his views have taken hold. The final recommendation was to make the squat parallel. This information was published in expert journals and was taken over by various health institutions or the military, which made this information even more popular. Unfortunately, these recommendations also appeared in textbooks and professional books and were therefore considered to be true. Already in the 1960s, coaching authorities publicly opposed it, but in vain. For many decades, such a deep squat came to a black list (Todd, 1984). Although many studies emerged that contradicted this fact, a reversal occurred in the 21st century (Myer et al., 2014). 
An important factor in the squat includes compression forces acting on the patellofemoral joint. According to the research by Escamilla (Escamilla, 2001), values increase proportionally with flexion of the knee and reach a peak at $130^{\circ}$. This fact is associated with possible deformation consequences when performing the deep squat. It is necessary to emphasize that the review lists studies that both use a mathematical model and did not directly measure. An analytical model can be used to calculate the effect of patellofemoral forces, taking into account all squat variables - femur and tibia length, patellar tendon length, body weight vector, or segmental angles (Fekete et al., 2014).

Mathematical models do not take into account the change of contact/friction surfaces in the knee or the function of the knee as a dynamic system, where the forces are distributed over all parts (Hehne, 1983). The neglect of rotational movements in the transverse plane (approximately $12^{\circ}$ ), which occur during the ascending phase of squatting, is also viewed critically. Natural internal rotation and adduction also influence the resulting action of forces (Kapandji, 2009). However, the claims of Escamilla (2001) do not coincide with Ahmed (Ahmed et al., 1983) or Hehne (Hehne, 1990), where different conclusions are drawn from the forces. To explain the measured values, it is important to realize that in any movement some forces act on soft structures or joints. An example is a push-up, where the wrist is also loaded, and it is not necessary to treat it as a risky matter (Polovinets et al., 2018).

\section{Negative effects of squatting}

Unfortunately, there are no studies on the long-term effects of the squat on the entire knee structure. A study by Kujala et al. (1995), which examined knee osteoarthritis in various sports. The results of weightlifters, where frequent use of deep squats can be expected, were comparable to long-distance runners. Thus, it can be concluded that the "wear and tear" is caused by excessive exercise load, not by a specific exercise. These are confirmed by Driban et al. (2015) who associate arthritis most with the sports of football, wrestling, weightlifting, American football, long distance running. An extreme case of using a deep squat (on tiptoe) is baseball, specifically the position of the catcher. The athlete will do a lot of squats during the match and also have to endure them for some time. This is subsequently associated with osteochondritis (McElroy et al., 2016). As with Olympic weightlifting or powerlifting, it is part of sports performance, which is a big difference from using squats as part of strength training or as an exercise for the general population.

There are situations where the use of deep squats must be limited or strictly adapted to the individual's state of health. One case is the cam morphology of the hip joint, which may be present without symptoms of impingement (Bagwell \& Powers, 2017). In these subjects, it is important to emphasize external hip rotation, enhanced extensors, and avoidance of large flexion (Catelli et al., 2018). In the presence of femoroacetabular impingement, an asymmetric design occurs, which must be avoided due to the automation of the movement pattern (Diamond et al., 2017). In general, good hip condition (muscle pain, fatigue, tightness) have a significant effect on squat performance and performance (Cheatham et al., 2017).

During squats, the ligaments stabilize the knee joint and thus have an important function. When they are injured or removed, the optimal technical design is affected (in the frontal and sagittal planes) and the resulting forces change not only in the knee but also in the entire locomotor system (Slater \& Hart, 2016). Free posterior cruciate ligament causes an increase in patellofemoral pressure (Shoifi Abudakar et al., 2016). In contrast, anterior cruciate ligament dysfunction tends to restrict forward knee movement (Stone et al., 2017).

As part of rehabilitation or prevention, various methods and aids are used to alleviate the forces acting on the knee joint. Orthoses are often used, which, when worn correctly, positively affect balance, coordination, proprioception, or muscle power (Baltaci et al., 2011). Knee sleeves 
have a similar function, although here the effect is amplified by subjective feeling (Sinclair et al. 2019). Knee wraps are also used in power sports, but they are not recommended for the general public or frequent use. In addition to increased discomfort, they cause a change in squat kinematics and may reduce the work of the vastus lateralis (Gomes et al., 2015). In contrast, kinesio taping can be recommended, which is a suitable method of knee fixation that does not alter muscle activation and brings a feeling of knee stability (Sarrão et al., 2016). "Knee support" or "knee savers" are used to mitigate the negative effects of deep squat positions on the tiptoes of baseball players (catchers). Their application is functional: they reduce the tension of the knee joint, reduce the forward movement of the knee, reduce the speed of the ascending phase (Doodley et al., 2019). However, it is a specific tool that is used only in this sport.

\section{CONCLUSION}

Deep squat has been associated for centuries with exercises that develop strength, fitness, and health. There are opinions supported primarily by historically older studies on the potential hazards related to knee instability or by theoretical-analytical models proclaiming overloading of the patellofemoral joint. However, such conclusions do not coincide with relevant studies. With optimal technique and sensible exercise selection, there is no greater risk in healthy individuals without degenerative anatomical changes. In order to assess the effects of the squat forces on the ankle, knee, hip, spine, it is necessary to take into account the technique and also individual anatomical differences. The knee joint is naturally adapted to move to a full squat and nothing prevents its use. Of course, with repeated and long-lasting high loads exceeding the adaptation potential, wear and tear associated with irreversible changes can occur. Functional aids are available for prevention or prophylaxis when the knee is subjected to deep squats.

Funding: This research received no external funding.

Conflicts of Interest: The authors have no conflicts of interest to declare.

\section{References}

AHMED, A. M. et al. (1983) In-vitro measurement of static pressure distribution in synovial joints--Part II: Retropatellar surface. Journal of Biomechanical Engineering. 105 (3), 226-236.

BAGWELL, J. J. \& POWERS, C. M. (2017) The Influence of Squat Kinematics and Cam Morphology on Acetabular Stress. Arthroscopy: The Journal of Arthroscopic \& Related Surgery: Official Publication of the Arthroscopy Association of North America and the International Arthroscopy Association. [Online] 33 (10), 1797-1803.

BALTACI, G. et al. (2011) The effect of prophylactic knee bracing on performance: balance, proprioception, coordination, and muscular power. Knee surgery, sports traumatology, arthroscopy: official journal of the ESSKA. [Online] 19 (10), $1722-1728$.

BEAUJEU, J. A. (1828) A treatise on gymnastic exercises: Or, Calisthenics for the use of young ladies : introduced at the Royal Hibernian Military School, also at the Seminary for the edication of young ladies. R. Milliken.

BISCARINI, A. et al. (2014) Voluntary enhanced cocontraction of hamstring muscles during open kinetic chain leg extension exercise: its potential unloading effect on the anterior cruciate ligament. The American Journal of Sports Medicine. [Online] 42 (9), 2103-2112.

CATELLI, D. S. et al. (2018) Asymptomatic Participants With a Femoroacetabular Deformity Demonstrate Stronger Hip Extensors and Greater Pelvis Mobility During the Deep Squat Task. Orthopaedic Journal of Sports Medicine. [Online] 6 (7), 2325967118782484.

CHEATHAM, S. W. et al. (2018) Hip Musculoskeletal Conditions and Associated Factors That Influence Squat Performance: A Systematic Review. Journal of Sport Rehabilitation. [Online] 27 (3), 263-273.

CONTRERAS, B. et al. (2015) A Comparison of Gluteus Maximus, Biceps Femoris, and Vastus Lateralis Electromyographic Activity in the Back Squat and Barbell Hip Thrust Exercises. Journal of Applied Biomechanics. [Online] 31 (6), $452-458$.

COX, C. F. \& BORDONI, B. (2019) 'Anatomy, Bony Pelvis and Lower Limb, Knee Posterior Cruciate Ligament', in StatPearls. Treasure Island (FL): StatPearls Publishing. p. [online]. Available from: http://www.ncbi.nlm.nih.gov/books/NBK535416/ (Accessed 1 June 2019). 
STUDIA SPORTIVA $2021 / 1$

DIAMOND, L. E. et al. (2017) Squatting Biomechanics in Individuals with Symptomatic Femoroacetabular Impingement. Medicine and Science in Sports and Exercise. [Online] 49 (8), 1520-1529.

DOOLEY, E. et al. (2019) The effects of knee support on the sagittal lower-body joint kinematics and kinetics of deep squats. Journal of Biomechanics. [Online] 82164-170.

DRIBAN, J. B. et al. (2017) Is Participation in Certain Sports Associated With Knee Osteoarthritis? A Systematic Review. Journal of Athletic Training. [Online] 52 (6), 497-506.

ESCAMILLA, R. F. et al. (2009) Cruciate ligament force during the wall squat and the one-leg squat. Medicine and Science in Sports and Exercise. [Online] 41 (2), 408-417.

ESCAMILLA, R. F. (2001) Knee biomechanics of the dynamic squat exercise. Medicine and Science in Sports and Exercise. 33 (1), 127-141.

ESCAMILLA, R. F. et al. (2008) Patellofemoral compressive force and stress during the forward and side lunges with and without a stride. Clinical Biomechanics (Bristol, Avon). [Online] 23 (8), 1026-1037.

FEKETE, G. et al. (2014) Patellofemoral Model of the Knee Joint Under Non-Standard Squatting. DYNA. [Online] 81 (183), 60-67.

FRY, A. C. et al. (2003) Effect of knee position on hip and knee torques during the barbell squat. Journal of strength and conditioning research. [Online] 17 (4), 629-633.

FUGLSANG, E. I. et al. (2017) Effect of Ankle Mobility and Segment Ratios on Trunk Lean in the Barbell Back Squat. Journal of Strength and Conditioning Research. [Online] 31 (11), 3024-3033.

GIESSING, J. \& TODD, J. (2005) The Origins of German Bodybuilding: 1790-1970. Iron Game History: The Journal of Physical Culture. 9 (2), 13.

GOMES, W. A. et al. (2015) Kinematic and sEMG Analysis of the Back Squat at Different Intensities With and Without Knee Wraps. Journal of Strength and Conditioning Research. [Online] 29 (9), 2482-2487.

GRZELAK, P. et al. (2012) Hypertrophied cruciate ligament in high performance weightlifters observed in magnetic resonance imaging. International Orthopaedics. [Online] 36 (8), 1715-1719.

HARTMANN, H. \& WIRTH, K. (2014) Literaturbasierte Belastungsanalyse unterschiedlicher Kniebeugevarianten unter Berücksichtigung möglicher Überlastungsschäden und Anpassungseffekte. Schweizerische Zeitschrift fur Sportmedizin und Sporttraumatologie. 626-23.

HEHNE, H. J. (1990) Biomechanics of the patellofemoral joint and its clinical relevance. Clinical Orthopaedics and Related Research. (258), 73-85.

HEHNE, H.-J. (1983) Das Patellofemoralgelenk: funktionelle Anatomie - Biomechanik - Chondromalazie u. operative Therapie. Enke.

JAHN, F. L. (1816) Die deutsche Turnkunst. Selbstverl.

KAPANDJI, I. A. (2009) Funktionelle Anatomie der Gelenke: schematisierte und kommentierte Zeichnungen zur menschlichen Biomechanik ; einbändige Ausgabe - obere Extremität, untere Extremität, Rumpf und Wirbelsäule. Georg Thieme Verlag.

KLEIN, K. (1959) A preliminary study of the dynamics of force as apllied to knee injury in athletics and as related to the supportting strength of the involved musculature. Medicina Sportive. 13 (7), 327-334.

KLEIN, K. (1961) The deep squat exercise as utilized in weight training for athletes and its effects on the ligaments of the knee. Journal of the Association for Physical and Mental Rehabilitation. 156-11.

KRAUSE, D. A. et al. (2015) Reliability and Accuracy of a Goniometer Mobile Device Application for Video Measurement of the Functional Movement Screen Deep Squat Test. International Journal of Sports Physical Therapy. 10 (1), 37-44.

KRITZ, M. et al. (2009) The Bodyweight Squat: A Movement Screen for the Squat Pattern. Strength \& Conditioning Journal. [Online] 31 (1), 76.

KUJALA, U. M. et al. (1995) Knee osteoarthritis in former runners, soccer players, weight lifters, and shooters. Arthritis and Rheumatism. [Online] 38 (4), 539-546.

KUSHNER, A. M. et al. (2015) The Back Squat Part 2: Targeted Training Techniques to Correct Functional Deficits and Technical Factors that Limit Performance. Strength and Conditioning Journal. [Online] 37 (2), 13-60.

LI, G. et al. (2004) Kinematics of the knee at high flexion angles: an in vitro investigation. Journal of Orthopaedic Research: Official Publication of the Orthopaedic Research Society. [Online] 22 (1), 90-95.

LOGTERMAN, S. L. et al. (2018) Posterior Cruciate Ligament: Anatomy and Biomechanics. Current Reviews in Musculoskeletal Medicine. [Online] 11 (3), 510-514.

MCELROY, M. J. et al. (2018) Catcher's Knee: Posterior Femoral Condyle Juvenile Osteochondritis Dissecans in Children and Adolescents. Journal of Pediatric Orthopedics. [Online] 38 (8), 410-417.

MYER, G. D. et al. (2014) The back squat: A proposed assessment of functional deficits and technical factors that limit performance. Strength and Conditioning Journal. [Online] 36 (6), 4-27.

POLOVINETS, O. et al. (2018) Force transmission through the wrist during performance of push-ups on a hyperextended and a neutral wrist. Journal of Hand Therapy: Official Journal of the American Society of Hand Therapists. [Online] 31 (3), 322-330.

RAMIREZ, M. et al. (2018) Quantifying Frontal Plane Knee Kinematics in Subjects with Anterior Knee Pain: the Reliability and Concurrent Validity of 2D Motion Analysis. International Journal of Sports Physical Therapy. 13 (1), 86-93.

SANDOW, E. (1894) Sandow on Physical Training: A Study in the Perfect Type of the Human Form. Gale \& Polden, Limited.

SCHOENFELD, B. J. (2010) Squatting kinematics and kinetics and their application to exercise performance. Journal of Strength and Conditioning Research. [Online] 24 (12), 3497-3506. 
SERRÃO, J. C. et al. (2016) Effect of 3 Different Applications of Kinesio Taping Denko ${ }^{\circledR}$ on Electromyographic Activity: Inhibition or Facilitation of the Quadriceps of Males During Squat Exercise. Journal of Sports Science \& Medicine. 15 (3), 403-409.

SHOIFI ABUBAKAR, M. et al. (2016) Influence of Posterior Cruciate Ligament Tension on Knee Kinematics and Kinetics. The Journal of Knee Surgery. [Online] 29 (8), 684-689.

SINCLAIR, J. et al. (2019) Acute effects of knee wraps/sleeve on kinetics, kinematics and muscle forces during the barbell back squat. Sport Sciences for Health. [Online]. Available from: https://doi.org/10.1007/s11332-019-00595-5 (Accessed 7 May 2020).

SLATER, L. V. \& HART, J. M. (2016) The influence of knee alignment on lower extremity kinetics during squats. Journal of Electromyography and Kinesiology: Official Journal of the International Society of Electrophysiological Kinesiology. [Online] 3196-103.

STONE, W. J. et al. (2017) Lower Extremity Kinematics of ACL - Repaired and Non-Injured Females when Using Knee Savers ${ }^{\circledast}$. International Journal of Sports Physical Therapy. 12 (5), 737-746.

TODD, J. \& HEMME, F. (2013) Florian Hemme and Jan Todd, "Beyond the Hack Squat: George Hackenschmidt's Forgotten Legacy as a Strength-Training Pioneer". Iron Game History: The Journal of Physical Culture. 123-18.

TODD, T. (1984) Historical Opinion: Karl Klein and the Squat. Strength \& Conditioning Journal. 6 (3), 26.

TOUTOUNGI, D. E. et al. (2000) Cruciate ligament forces in the human knee during rehabilitation exercises. Clinical Biomechanics (Bristol, Avon). 15 (3), 176-187.

WALSH, J. C. et al. (2007) Three-dimensional motion analysis of the lumbar spine during 'free squat' weight lift training. The American Journal of Sports Medicine. [Online] 35 (6), 927-932.

WILK, K. E. et al. (1996) A comparison of tibiofemoral joint forces and electromyographic activity during open and closed kinetic chain exercises. The American Journal of Sports Medicine. [Online] 24 (4), 518-527.

YAVUZ, H. U. \& Erdag, D. (2017) Kinematic and Electromyographic Activity Changes during Back Squat with Submaximal and Maximal Loading. Applied Bionics and Biomechanics. [Online] 20179084725. 